\title{
DISTURBED RADIO EMISSION FROM \\ THE SUN AS A SUM OF SMALL MONOGHROMATIG PEAKS
}

\author{
V. V. VITKEVITCH \\ Crimean Station, Physical Institute of the Academy of Sciences, Moscow, U.S.S.R.
}

Observations of the radio emission from the sun carried out during recent years at the Crimean Station of the Physical Institute of the U.S.S.R. Academy of Sciences showed that the occurrence of spots appreciably increases the intensity of the solar radio emission in the range of metre wave-lengths. This increase of intensity has two components. The first ( $S$-component) changes comparatively slowly with time. The second ( $P$-component) consists of individual brief bursts (of the order of a second and less) of small amplitudes (10-100 \% of the intensity of the quiet sun). The $P$-component is manifested most clearly in the emission connected with spots of small areas, when the general increase of the intensity is insignificant. Such a situation has been utilized for the study of the spectrum of individual small peaks.

Observations were carried out as follows [1]. Two radio receivers, adjusted for frequencies differing by $4 \mathrm{Mc}$./s., were connected successively to the aerial with ninety-six half-wave dipoles with a reflector. As the band-width of the aerial equalled 4-5 Mc./s., radio waves were received reliably by the two receivers. The intensity of the radio emission from the sun for the two wave-lengths was recorded at the output terminals of each of the two receivers. The intensities of the peaks that appeared simultaneously on the two frequencies were compared. A statistical method was applied in order to determine the effective width of the spectra of individual peaks. It was found that the band-width is on the average $6 \mathrm{Mc} . / \mathrm{s} .=0 \cdot 029 f_{0}$ for peaks of small amplitude and I I Mc./s. $=0.054 f_{0}$ for peaks of large amplitude. This corresponds to values of $Q=f_{0} / \Delta f=35$ and 18 , respectively.

These preliminary mean values characterize the radio emission spectrum 363 
of the peak component of the sun for $1 \cdot 5$ metres wave-length. It is seen that the bands in which the peaks are generated are extremely narrow. The radio emission of separate peaks thus is of a monochromatic nature. Similar results were obtained by other authors [2].

2

A number of observations of the radio emission from the peaks was carried out by means of a multi-channel radio-spectrograph. The multichannel radio-spectrograph has the advantage over the Australian radiospectrograph of discrete bands for the reception of the radio waves. Records are obtained at all channels simultaneously. The sensitivity of our radio-spectrograph is much higher than that of the Australian so that the separate peaks of radio emission from the sun are observed quite distinctly.

A comparison of the intensities of the peaks occurring in neighbouring wave-lengths in the range of 2-4 metres, in a similar manner as was used for the 1.5 waves, showed that the band-width of the peaks is again extremely narrow, of the order of 4-7 Mc./s.

Thus it may be concluded that the narrow band in which a peak is generated is a common feature for radio waves in the range of metre wavelengths. The duration of small peaks for radio waves of 3-4 metres is somewhat longer than for radio waves of $\mathrm{I} \cdot 5$ metres.

Observations have shown that, if peaks occur continually at some wavelength of the metre range, they are also observed at other wave-lengths.

Cases were met when peaks were not observed on a wave-length $\lambda_{0}$ and all larger wave-lengths, while they were noticed in shorter waves. Thus, the peaks seem to break off abruptly somewhere in the region of shorter wave-lengths, being absent on larger wave-lengths. The value $\lambda_{0}$ often lies in the range of 2.5-3 metres. A detailed investigation of the range of wave-lengths where the peaks are generated is extremely important and should be carried out in detail.

Daily observations of the radio emission from the sun on $\mathrm{I} \cdot 5$ metres have been carried out by us since 1953 . Observations were carried out during I or $2 \mathrm{hr}$. per day in the periods when the radio emission was of a quiet nature, and in addition during some hours without interruption, when the radio emission had a disturbed character.

During days of disturbed radio emission, a general increase of the radio emission from the sun was always accompanied by the occurrence of small 
peaks. Their duration averaged $\mathrm{I}-2$ sec. We paid particular attention to the relation between the $P$ - and $S$-components. We shall define the value $S$ as the increase of the intensity of the continuous radio emission, and the value $P$ as the mean intensity of the peaks. It is convenient to express both values in units of the radio emission from the quiet sun during subsequent days. It was discovered that the $P$-component may occur in the absence of the $S$-component. But the $S$-component is never present unless the $P$-component is there. It follows that a rise of the intensity is always connected with the presence of peaks.

A further study of this question showed that whenever the $S$-component is present the peaks follow each other without interruption and no cases when peaks were absent in the presence of the $S$-component were observed. As soon as one peak disappears another one, superimposed upon the foregoing peak, originates. It is possible to conclude from this fact that the component of the disturbed radio emission from the sun connected with spots and flocculi is the sum of monochromatic peaks of radio emission, unresolved in time.

An increase of the intensity of radio emission not connected with the occurrence of peaks was noticed only once in the course of the entire period of observation. Such cases must apparently be considered as exceptional and their observation is of particular importance.

\section{REFERENCES}

[1] Vitkevitch, V. V. Dokl. Akad. Nauk U.S.S.R. 1or, 229, 1955.

[2] Blum, E. Ann. Univ. Paris, 23, no. 1, 136, 1953.

\section{Discussion}

Smith: Very fast recordings with a time constant of $o \cdot I$ sec. made in Cambridge at $\lambda=\mathrm{I} \cdot 4$ metres, in 1949 and $195^{\circ}$, showed the presence of a steady component with superimposed bursts often well separated in time.

Owren: Recordings with a time constant of o.or sec. made at Cornell University at $200 \mathrm{Mc}$./s. since $195^{\circ}$ fully confirm this. 